\title{
Adult-onset Very Long-Chain Acyl-CoA Dehydrogenase Deficiency (VLCADD)
}

\author{
Farzad Fatehi, M.D., M.S.c ${ }^{1}$, Ali Asghar Okhovat, M.D. ${ }^{1}$, Yalda Nilipour, M.D. ${ }^{2,3}$, Magdalena \\ Mroczek, M.D. ${ }^{4}$, Volker Straub, M.D., PhD. ${ }^{4}$, Ana Töpf, PhD. ${ }^{4}$, Aleksa Palibrk, M.D. ${ }^{5}$, Stojan \\ Peric, M.D. ${ }^{5}$, Vidosava Rakocevic Stojanovic, M.D. ${ }^{5}$, Hossein Najmabadi ${ }^{6}$, Shahriar Nafissi, \\ M.D. ${ }^{1}$ \\ ${ }^{1}$ Neurology Department, Shariati Hospital, Tehran University of Medical Sciences, Tehran, Iran \\ 2Pediatric Pathology Research Center, Research Institute for Children's Health, Shahid Beheshti \\ University of Medical Sciences, Tehran, Iran \\ ${ }^{3}$ Mofid Children Hospital, School of Medicine, Shahid Beheshti University of Medical Sciences, \\ Tehran, Iran \\ 4John Walton Muscular Dystrophy Research Centre, Translational and Clinical Research Institute, \\ Newcastle University and Newcastle Hospitals NHS Foundation Trust, Newcastle upon Tyne, UK \\ ${ }^{5}$ Neurology Clinic, Clinical Center of Serbia, Faculty of Medicine, University of Belgrade, Belgrade, \\ Serbia \\ ${ }^{6}$ Genetics Research Center, University of Social Welfare and Rehabilitation Sciences, Tehran, \\ Iran
}

\section{Abstract}

Background-Very long-chain acyl-CoA dehydrogenase deficiency (VLCADD) is a hereditary disorder of mitochondrial long-chain fatty acid oxidation that manifests with variable presentations, including exercise intolerance, cardiomyopathy, and liver disease. Herein, we describe the clinical and genetic manifestations of six patients with adult-onset VLCADD.

Methods-In this study, clinical, pathological, and genetic findings of six adult patients (four Iranian and two Serbian) with VLCADD and their response to treatment are described.

Results-The median age of patients at first visit was 31 (range: 27-38) years, and the median age of onset was 26.5 (range: 19-33) years. Parental consanguinity was present for four patients. Four patients had a history of rhabdomyolysis, and the recorded CK ranged between 67 and 90,000 IU/L. Three patients had a history of exertional myalgia, and one patient had a nonfluctuating weakness.

\footnotetext{
*Corresponding Author: Shahriar Nafissi, MD, Professor of Neurology, Neuromuscular Department, Shariati Hospital, Tehran University of Medical Sciences, Iran.nafisi@tums.ac.ir, nafissishahriar@gmail.com.

Disclosure

The authors report no disclosures relevant to the manuscript.

Conflict of interest: the authors declare no conflict of interest.
} 
Through next-generation sequencing analysis, we identified six cases with variants in the ACADVL gene and a confirmed diagnosis of VLCADD. Of the total six variants identified, five were missense, and one was a novel frameshift mutation identified in two unrelated individuals. Two variants were novel, and three were previously reported. We treated the patients with a combination of L-Carnitine, CoQ10, and Riboflavin. Three patients responded favorably to the treatment.

Conclusion-Adult-onset VLCADD is a rare entity by various presentations. Patients may respond favorably to a cocktail of L-Carnitine, Coenzyme Q, and Riboflavin.

\section{Keywords}

Very Long-Chain Acyl-CoA Dehydrogenase; VLCAD Deficiency; Lipid Storage Myopathy; Metabolic Myopathy; Neuromuscular diseases; Inherited metabolic disorders

\section{Introduction}

Metabolic myopathies encompass a clinically and etiologically heterogeneous group of disorders affected by deficiencies in cellular energy metabolism comprising the breakdown of carbohydrates and fatty acids to generate adenosine triphosphate, predominantly through mitochondrial oxidative phosphorylation [1]. Very long-chain acyl-CoA dehydrogenase deficiency (VLCADD), OMIM 609575, is a hereditary disorder of mitochondrial long-chain fatty acid oxidation that manifests with variable presentations including exercise intolerance and rhabdomyolysis, cardiomyopathy, liver disease, and hypoketotic hypoglycemia [2]. Very long-chain acyl-CoA dehydrogenase is bound to the inner membrane of mitochondria and catalyzes the initial phase of the long-chain fatty acid $\beta$-oxidation [3]. This mitochondrial enzyme is the first enzyme in the fatty acid oxidation cycle and, as such, a crucial enzyme in this pathway for mitochondrial energy transduction from fatty acids [4]. The mode of inheritance of VLCADD is autosomal recessive, and in keeping with the age at onset of clinical manifestations, three phenotypes have been described: (1) a severe type starting in infancy manifesting with liver failure and hypertrophic cardiomyopathy; (2) a childhoodonset form initiating between ages 1 and 13 years presenting with hypoketotic hypoglycemia; and (3) a juvenile or adult-onset muscular form characterized by recurring bouts of rhabdomyolysis generated by prolonged activity or fasting [5]. Herein, we describe the clinical and genetic manifestations of six adult patients with VLCADD, four from Iran, and two from Serbia. Some patients had manifestations that had not previously described in VLCADD, such as non-fluctuating weakness and isolated hyper-CKemia. It is noticeable that in the initial approach, the diagnosis was other than VLCADD.

\section{Material and Methods}

This study was performed in Shariati Hospital affiliated to Tehran University of Medical Sciences and Neurology Clinic, Clinical Center of Serbia, University of Belgrade. The patients were enrolled between the years 2015 and 2019.

Initially, the patients had been visited for a combination of fluctuating muscle weakness (except patient 1), exercise intolerance, rhabdomyolysis, or high creatine kinase (CK), and a 
primary diagnosis of metabolic myopathy had been made. Subsequently, muscle biopsy or directly Next-generation sequencing was recommended. The ultimate diagnosis of VLCADD was made based on the genetic study and its correlation with the patients' phenotype.

We collected information such as age at the first visit, age of onset, gender, ethnicity (Persian or Serbian), consanguinity, creatine kinase level [CK (IU/L)] (normal range between 20 and $200 \mathrm{IU} / \mathrm{L}$ ), creatinine level during rhabdomyolysis (normal range between 0.6 and $1.2 \mathrm{mg} / \mathrm{dL}$ ), cardiac involvement, and the history of rhabdomyolysis.

For clinical evaluation, we assessed Manual Muscle Testing (MMT) using the 6-point Medical Research Council (MRC) scale (range 0-5). Also, we evaluated the functional scale of Brooke [6] for upper extremities (range: 1-6) and Vignos[7] for lower extremities (range: $1-10)$.

For three patients (Patients 1, 4, 5), open muscle biopsy had been performed before the ultimate diagnosis as a part of the diagnostic approach for myopathies. The biopsies were conducted when the rhabdomyolysis had been resolved.

For patient 1 , we performed a targeted next-generation sequencing panel (NGS) for metabolic myopathies, and for other patients, whole-exome sequencing was recommended. For patients 3, 5, and 6, whole-exome sequencing analysis was conducted through the MYO-SEQ project [8]). MYO-SEQ project is an international research collaboration that applied targeted whole-exome sequencing (WES) to around 1000 patients with undiagnosed proximal muscle weakness [8]. DNA samples were submitted to the Newcastle MRC Centre Biobank for Neuromuscular Diseases for which ethical approval was granted by the NRES Committee North East - Newcastle \& North Tyneside 1 (reference 08/H0906/28). All patients gave informed consent before the study enrolment.

\section{In silico predictions}

Variant pathogenicity predictions were completed using deleteriousness prediction methods for single-nucleotide variants in whole-exome sequencing studies such as Polyphen2_HDIV[9], Mutation Taster[10], FATHMM[11], PROVEAN[12], and CADD[13].

\section{Data availability}

Anonymized data not published within this article will be made available by request from any qualified investigator.

\section{Results}

The clinical and genetic manifestations of six adult patients with VLCADD are summarized in Table 1.

The median age of patients at first visit was 31 (range: 27-38) years, and the median age of onset was 26.5 (range: 19-33) years. Two patients were male (patients 1,2), and parental consanguinity was present for four patients (patients 1-4). Four patients had a history of 
rhabdomyolysis (patients 1-3 and 5), and the recorded CK ranged between 67 and 90,000 IU/L. Three patients had a history of exertional myalgia (patients 1-3), and one patient had a non-fluctuating weakness (patient 1).

In Silico prediction, analyses are described in the Supplementary Table. In Polyphen2_HDIV analysis, p.Met300Ile, and p.Met437Thr demonstrated possible damaging whereas other variants demonstrated probable damaging. In Mutation Taster all variants were disease-causing. In FATHMM, all variants were damaging, and in PROVEAN, all were deleterious.

Herein, the detailed history of patients is described:

\section{Patient 1}

A 38-year-old Iranian man was presented with slowly progressive, lower limb weakness since the age of 19 years. The patient was unable to climb stairs since the age of 36 . In addition, he described episodes of myalgia and dark urine following exercise. The parents were first cousins, with no similar presentation in the family (Figure 1). He had weakness of neck flexion (MRC grade 3), arms abduction (MRC grade 3), elbow flexion (MRC grade 4), hip flexion, and knee extension (MRC grade 4). The functional scale of Brooke[6] for upper extremities was 3 (out of 6), and the functional scale of Vignos[7] for lower extremities was 4 (out of 10).

Creatine kinase (CK) level was up to $5400 \mathrm{IU} / \mathrm{L}$, and needle electromyography (EMG) showed myopathic changes in proximal muscles of upper and lower limbs without spontaneous activity. Cardiac exams, including physical examination, electrocardiogram, and echocardiography, were normal. Muscle MRI of the thigh and lower legs disclosed moderate deposition of fat in adductor, and vastus muscles as well as medial heads of gastrocnemius muscles (Figure 2A). Muscle biopsy from left deltoid revealed atrophy of mainly type 1 fibers with multiple necrotic and degenerative/regenerative fibers, some nuclear clumps, and no endomysial fibrosis. Oil Red O stain showed fine lipid droplets in muscle fibers, and cytochrome oxidase + succinate dehydrogenase stain (COX+SDH) revealed some COX-negative fibers (Figure 3a-c). Targeted next-generation sequencing (NGS) for metabolic myopathies revealed a novel homozygous pathogenic variant (NM_000018.4:c.900G>A; p.(Met300Ile)) in exon 10 of the $A C A D V L$ gene, confirming the diagnosis of VLCADD. The same variant in heterozygous form was seen in both unaffected parents.

We started L-Carnitine 2 grams daily, Coenzyme Q $300 \mathrm{mg}$ daily, and Riboflavin $300 \mathrm{mg}$ daily with a favorable response. After two months, the patient could climb stairs without aid. The functional scales of Brooke and Vignos were both one, and the CK reduced to $320 \mathrm{IU} / \mathrm{L}$.

\section{Patient 2}

A 27-year-old Iranian man complained of muscle weakness and exercise intolerance with ensuing severe weakness after exercise from the age of 23 years. One month before the visit, the patient experienced dark brown urine after a long walk, followed by muscle weakness. Subsequently, he developed elevated blood urea nitrogen and creatinine (serum creatinine 
$11 \mathrm{mg} / \mathrm{dL}$ ), and the CK was $25000 \mathrm{IU} / \mathrm{L}$. Hemodialysis had been started, and muscle strength improved over several weeks. Parents were first cousins, and family history was negative (Figure 1). On examination, he had symmetrical weakness of neck flexion, arm abduction (MRC grade 4), and hip flexion (MRC grade 4) with normal deep tendon reflexes. The functional scale of Brooke for upper extremities was 2 (out of 6), and the functional scale of Vignos for lower extremities was 2 (out of 10). After one month, CK was 14180 IU/L; cardiac and respiratory evaluations were normal, and electromyography was myopathic in proximal muscles of upper and lower limbs. Muscle MRI of thigh and legs revealed moderate fat deposition in hamstrings and sartorius muscles, as well as medial heads of gastrocnemius and soleus muscles. Whole-exome sequencing revealed a homozygous pathogenic variant (NM_000018.4:c.911C > T; p.(Ala304Val)) in exon 10 of the $A C A D V L$ gene. We started L-Carnitine 2 grams daily, Coenzyme Q 300 mg daily, and Riboflavin 300 $\mathrm{mg}$ daily with a favorable response and $\mathrm{CK}$ reduced to below 1,000 IU/L within six months. The functional scales of Brooke and Vignos were both one after six months.

\section{Patient 3}

This 27-year-old Iranian woman referred to the emergency department without a history of previous illness, with muscle weakness of four extremities starting three days before. She reported a previous episode of dark urine after prolonged exercise one year before. The parents were second cousins. Two brothers, one at the age of 3 years and the other one at the age of 4 years, and her sister died of cardiomyopathy at the age of 10 months; she also had an older sister and three younger brothers who were healthy. On examination, she had neck flexor weakness, bilateral arms abduction (MRC grade 2), bilateral elbow flexion (MRC grade 3), bilateral hip flexion (MRC grade 2), and bilateral knee extension (MRC grade 3) with normal deep tendon reflexes. The functional scale of Brooke for upper extremities was 5 (out of 6), and the functional scale of Vignos for lower extremities was 9 (out of 10). The CK was $46000 \mathrm{IU} / \mathrm{L}$ during admission; echocardiography was normal, and electromyography was myopathic in proximal and distal muscles of upper and lower extremities with prominent spontaneous activity. Subsequently, the patient developed acute kidney injury (serum creatinine $8.2 \mathrm{mg} / \mathrm{dL}$ ), and hemodialysis introduced. Muscle strength improved over several weeks. Targeted whole-exome sequencing analysis through the MYOSEQ project [8] revealed two compound heterozygous variants: a novel frameshift in exon 9 (NM_000018.4:c.869del; p.(Gly290Alafs*63)) and a previously reported missense variant in exon 11 (NM_000018.4:c.1097G>A; p.(Arg366His))[14]) which were confirmed with Sanger Sequencing. L-Carnitine, Riboflavin, and Coenzyme Q in reported doses were started. The functional scales of Brooke and Vignos were both one after six months, and the CK had declined to $380 \mathrm{IU} / \mathrm{L}$.

\section{Patient 4}

A 31-year-old Iranian woman without a history of any medical disorder was evaluated due to elevated liver enzymes (AST, 102 IU/L; ALT, 122 IU/L) and high CK (7057 IU/L) for preemployment checkups. She did not report any exercise intolerance or weakness. Her parents were first cousins, and she had two asymptomatic older sisters. On neurological examination, muscle power was normal, and electromyography and echocardiography were normal as well. Muscle MRI of thigh and legs revealed mild fat deposition in gastrocnemius 
and soleus muscles (Figure 2B). Muscle biopsy from the lateral head of right gastrocnemius revealed mixed myopathic and neurogenic atrophy. Groups of angular atrophic fibers were seen associated with dispersed necrotic fibers and prominent fiber type grouping. A slight increase in the lipid content of some muscle fibers was also noted (Figure $3 \mathrm{~d}-\mathrm{g}$ ). Wholeexome sequencing revealed compound heterozygous pathogenic variants in the $A C A D V L$ gene: a novel frameshift in exon 9 (NM_000018.4:c.869del; p.(Gly290Alafs*63)) and a previously reported missense variant in exon 13 (NM_000018.4:c.1310T>C; p.(Met437Thr)) [14], confirmed by Sanger sequencing.

We started treatment with L-Carnitine, Riboflavin, and Coenzyme Q. After 12 months, the CK did not significantly change (6584 IU/L).

\section{Patient 5}

The patient is a 31-year-old Serbian woman who was diagnosed with epilepsy at the age of 20. She took Levetiracetam and did not have any seizures during the last three years. At the age of 26 years, she noticed the presence of severe progressive fatigue during normal physical activities. CK level was 3180 IU/L, AST 344 (IU/L), and ALT 325 (IU/L). The cardiac evaluation was normal; forced vital capacity (FVC) was normal; however, maximum and minimum inspiratory pressures were $60 \%$ and $44 \%$, respectively. On neurological examination, there was mild weakness of facial muscles, flexor and extensor muscles of the neck, and the upper and lower extremities (MRC 4 in proximal muscles of upper and lower extremities, and MRC 3 in distal muscles of lower extremities). During exacerbations, she could not walk on her toes and heels and had difficulties getting up from a squatting position. Through the course of the disease, CK levels varied between 90 and 90,000 IU/L, paralleling clinical worsening. All muscle reflexes were diminished. Both parents were healthy and non-consanguineous. The functional scale of Brooke for upper extremities was 2 (out of 6), and the functional scale of Vignos for lower extremities was 3 (out of 10). Muscle MRI of lower limbs revealed fine fat deposition in gastrocnemius and soleus muscles (Figure 2C). Muscle biopsy indicated scarce inflammatory infiltrations and rare CD56+ lymphocytes in contact with the necrotic muscle fibers (Figure $3 \mathrm{~h}-\mathrm{i}$ ). Targeted whole-exome sequencing analysis through the MYO-SEQ project revealed a likely pathogenic homozygous missense variant (NM_000018.4:c.1358G>A; p.(Arg453Gln)), in exon 14 of the $A C A D V L$ gene, confirming the diagnosis of VLCADD. L-Carnitine 1 gram daily, Coenzyme Q $200 \mathrm{mg}$ daily, and Riboflavin $200 \mathrm{mg}$ daily were started with a favorable response. The functional scale of Brooke after treatment varied between 1 and 2, and the functional scale of Vignos ranged between 2 and 3 paralleling CK levels.

\section{Patient 6}

This patient is a 33-year-old Serbian woman and the sister of Patient 5. She complained of mild fatigue during normal physical activities. On neurological examination, she had mild proximal weakness in her legs (MRC 4+/5) and CK of $67 \mathrm{U} / \mathrm{L}$. Needle EMG showed spontaneous activities at rest and myopathic pattern during activation. Targeted wholeexome sequencing analysis through the MYO-SEQ project identified a likely pathogenic homozygous variant in the $A C A D V L$ gene, NM_000018.4:c.1358G>A; p. (Arg453Gln), the same as in her sister. 


\section{Discussion}

In this study, we described six patients with adult-onset VLCADD with variable presentations including frequent symptoms such as exercise intolerance (Patients 2, 3 and 5), rhabdomyolysis and myoglobinuria (Patients 1,2,3 and 5), and, to the best of our knowledge, not previously reported features such as non-fluctuating weakness (Patient 1), isolated hyper-CKemia (Patient 4), and mild fatigue and weakness with normal CK (Patient $6)$.

Reports of patients with adult-onset VLCADD remain infrequent. In the literature, nine cases of the genetically-proven adult-onset form of VLCADD have been described that started over the age of 16 years (Table 2)[15-23]. In previously reported patients, the mean age at onset of adult-onset VLCADD was $28 \pm 14$ years (range: 16-51 years), and the age at onset in our patients was slightly lower ( $25 \pm 4$ years) that resembles those previously described [15-23].

Common clinical manifestation in previously described patients included exercise intolerance and muscle pain after physical activity with rhabdomyolysis in severe cases [1523]. However, in one of our patients (Patient 1), there was a non-fluctuating weakness, and in another one (Patient 4), the clinical presentation was isolated hyper-CKemia, incredibly lacking either weakness or any episodes of exercise intolerance, which has not been reported in the literature so far. One of our patients (Patient 6), was paucisymptomatic with a normal CK despite myopathic EMG with spontaneous activities. The clinical picture of our patients demonstrated that, although most cases of adult-onset VLCADD may manifest as exercise intolerance and rhabdomyolysis, they can also present as non-fluctuating weakness or isolated hyper-CKemia.

Muscle MRI is non-specific in VLCADD, and one-third of patients with long-standing VLCADD, show mild proximal signal changes in T1-weighted sequences in the posterior compartment of the thigh and around 40\% in the posterior compartment of the leg [24]. In our series, only one patient with non-fluctuating weakness (Patient 1) showed noticeable signal changes on muscle MRI in the posterior compartment of thigh and leg, whereas all other patients displayed a faint signal change.

Muscle biopsy in VLCADD may show mild lipid accumulation in only around one-third of patients (Patients 1 and 4 in our series); hence, muscle biopsy may generally be inconclusive for the diagnosis of the disease; nevertheless, muscle biopsy in some patients might show mixed neurogenic and myopathic atrophies (Patient 4) resembling muscular dystrophies, or similar to inflammatory infiltrates in inflammatory myopathies (Patient 5) [25].

Consequently, normal lipid staining of muscle does not exclude VLCADD, and it should be vigilant for the mimickers of VLCADD on muscle biopsy.

Several mutations in the $A C A D V L$ gene have been reported (http://www.hgmd.cf.ac.uk/ac/ gene.php?gene=ACADVL). The most common variant found in the early-onset type is c.848T>C (p.Val283Ala) in exon 10 [26-28], which is previously described only in one patient with adult-onset type [21]; none of our patients had such mutation. In three of our patients (Patients 1, 3, and 4), we found novel variants; interestingly, Patients 3 and 4 who 
were none-related remarkably shared variant p.(Gly290Alafs*63) which is absent in the control population (https://gnomad.broadinstitute.org).

For the treatment of patients, the therapeutic strategies are controversial. In a Delphi clinical practice protocol for the management of VLCADD performed in 2009 [29], the panel recommended administering oral Carnitine only when needed to avert Carnitine deficiency in plasma. In patients with muscular symptoms such as pain and aching, a regimen with a medium-chain fat-rich meal directly before exercise may be beneficial [29]. L-Carnitine administration for patients with VLCADD is not generally accepted even though carnitine levels are low in the plasma of patients with VLCADD, causing a secondary carnitine deficiency [30]. However, some studies have shown that taking L-Carnitine not only prevents its secondary deficiency; but also it can prevent oxidative damage secondary to increased production of reactive species [31]. Nonetheless, our patients did not deteriorate with carnitine therapy, and even the non-fluctuating weakness of Patient 1 improved with the treatment.

One limitation of our study was that we did not check for the acylcarnitine profile since all patients were diagnosed after whole-exome sequencing. The measurement of plasma acylcarnitines by tandem mass spectrometry has been used for the diagnosis of VLCAD deficiency, showing an accumulation of long-chain acylcarnitines with tetradecenoylcarnitine (C14:1) as predominant acylcarnitine. Also, there can be a secondary deficiency of Carnitine. Stimulatingly, distinctive abnormal acylcarnitine profile is also observed in other fatty acid oxidation disorders such as mitochondrial trifunctional protein and multiple acyl-CoA dehydrogenase deficiencies, which may be manifested by muscle weakness or rhabdomyolysis in adults.

In conclusion, though most of the patients suffering from VLCADD, present in childhood, and often manifest as myoglobinuria and exercise intolerance, however, our patients presented at an older age, i.e., adult-onset form even in the third decade. In later-onset presentations, atypical manifestations such as non-fluctuating weakness and hyper-CKemia may be observed, and conspicuously the patients may be clinically asymptomatic. What is more, it seems that treatment with L-Carnitine may be an option for this rare form of lipid storage myopathy.

\section{Supplementary Material}

Refer to Web version on PubMed Central for supplementary material.

\section{Acknowledgment}

The analysis was provided by the Broad Institute of MIT and Harvard Center for Mendelian Genomics (Broad $\mathrm{CMG}$ ).

Study funding

Exome sequencing for patients 3, 5, and 6 was done through the MYO-SEQ project and was funded by Sanofi Genzyme, Ultragenyx, LGMD2I Research Fund, Samantha J Brazzo Foundation, LGMD2D Foundation, and Kurt + Peter Foundation, Muscular Dystrophy UK, and Coalition to Cure Calpain 3. The analysis was funded by the National Human Genome Research Institute, the National Eye Institute, and the National Heart, Lung, and Blood Institute grant UM1 HG008900 and in part by National Human Genome Research Institute grant R01 HG009141. 


\section{References}

1. Berardo A, DiMauro S, Hirano M. Diagnostic algorithm for metabolic myopathies. Curr Neurol Neurosci Rep. 2010; 10: 118-26. [PubMed: 20425236]

2. Leslie ND, Valencia CA, Strauss AW, Zhang K. Very Long-Chain Acyl-Coenzyme A Dehydrogenase Deficiency. 2018

3. Izai K, Uchida Y, Orii T, Yamamoto S, Hashimoto T. Novel fatty acid beta-oxidation enzymes in rat liver mitochondria. I. Purification and properties of very-long-chain acyl-coenzyme A dehydrogenase. J Biol Chem. 1992; 267: 1027-33. [PubMed: 1730632]

4. Diekman EF, Visser G, Schmitz JPJ, Nievelstein RAJ, de Sain-van der Velden M, Wardrop M, et al. Altered Energetics of Exercise Explain Risk of Rhabdomyolysis in Very Long-Chain Acyl-CoA Dehydrogenase Deficiency. PloS One. 2016; 11: e0147818. [PubMed: 26881790]

5. Laforêt P, Acquaviva-bourdain C, Rigal O, Brivet M, Penisson-besnier I, Chabrol B, et al. Neuromuscular Disorders Diagnostic assessment and long-term follow-up of 13 patients with Very Long-Chain Acyl-Coenzyme A dehydrogenase ( VLCAD ) deficiency. Neuromuscul Disord. 2009; 19: 324-9. [PubMed: 19327992]

6. Brooke MH, Griggs RC, Mendell JR, Fenichel GM, Shumate JB, Pellegrino RJ. Clinical trial in duchenne dystrophy. I. The design of the protocol. Muscle Nerve. 1981; 4: 186-97. [PubMed: 7017401]

7. VIGNOS PJ, ARCHIBALD KC. Maintenance of ambulation in childhood muscular dystrophy. $\mathbf{J}$ Chronic Dis. 1960; 12: 273-90. [PubMed: 13842210]

8. Johnson K, Töpf A, Bertoli M, Phillips L, Blain A, Ensini M, et al. The MYO-SEQ project: Application of exome sequencing technologies of 1000 patients affected by limb-girdle weakness of unknown origin. Neuromuscul Disord. 2017; 27: S194.

9. Adzhubei IA, Schmidt S, Peshkin L, Ramensky VE, Gerasimova A, Bork P, et al. A method and server for predicting damaging missense mutations. Nat Methods. 2010; 7: 248-9. [PubMed: 20354512]

10. Schwarz JM, Cooper DN, Schuelke M, Seelow D. MutationTaster2: mutation prediction for the deep-sequencing age. Nat Methods. 2014; 11: 361-2. [PubMed: 24681721]

11. Shihab HA, Gough J, Cooper DN, Stenson PD, Barker GLA, Edwards KJ, et al. Predicting the functional, molecular, and phenotypic consequences of amino acid substitutions using hidden Markov models. Hum Mutat. 2013; 34: 57-65. [PubMed: 23033316]

12. Choi Y, Sims GE, Murphy S, Miller JR, Chan AP. Predicting the Functional Effect of Amino Acid Substitutions and Indels. PLOS ONE. 2012; 7: e46688. [PubMed: 23056405]

13. Rentzsch P, Witten D, Cooper GM, Shendure J, Kircher M. CADD: predicting the deleteriousness of variants throughout the human genome. Nucleic Acids Res. 2019; 47: D886-94. [PubMed: 30371827]

14. Andresen BS, Olpin S, Poorthuis BJHM, Scholte HR, Vianey-Saban C, Wanders R, et al. Clear correlation of genotype with disease phenotype in very-long-chain acyl-CoA dehydrogenase deficiency. Am J Hum Genet. 1999; 64: 479-94. [PubMed: 9973285]

15. Herrera-Olivares AM, Fernández-Luque JA, Paradas C, Lucia A, Santalla A. Combined HIIT and resistance training in very long-chain acyl-CoA dehydrogenase deficiency: A case report. Front Physiol. 2019; 10: 650. [PubMed: 31191348]

16. Hisahara S, Matsushita T, Furuyama H, Tajima G, Shigematsu Y, Imai T, et al. A Heterozygous Missense Mutation in Adolescent-Onset Very Long-Chain Acyl-CoA Dehydrogenase Deficiency with Exercise-Induced Rhabdomyolysis. Tohoku J Exp Med. 2015; 235: 305-10. [PubMed: 25843429]

17. Voermans NC, Poels PJ, Kluijtmans LA, Engelen BG van. The effect of dantrolene sodium in Very Long Chain Acyl-CoA Dehydrogenase Deficiency. Neuromuscul Disord. 2005; 15: 844-6. [PubMed: 16288870]

18. Smelt AHM, Poorthuis BJHM, Onkenhout W, Scholte HR, Andresen BS, van Duinen SG, et al. Very long chain acyl-coenzyme A dehydrogenase deficiency with adult onset. Ann Neurol. 1998; 43: 540-4. [PubMed: 9546340] 
19. Very-Long-Chain Acyl-CoA Dehydrogenase Deficiency--diagnostic difficulties and own experience in multidisciplinary management. - PubMed - NCBI [Internet]. [cited 2019 Sep 6]. Available from: https://www.ncbi.nlm.nih.gov/pubmed/26982752

20. Murata K, Sugie H, Nishino I, Kondo T, Ito H. A primigravida with very-long-chain acyl-CoA dehydrogenase deficiency. Muscle Nerve. 2014; 49: 295-6. [PubMed: 23966064]

21. Mendez-Figueroa H, Shchelochkov OA, Shaibani A, Aagaard-Tillery K, Shinawi MS. Clinical and biochemical improvement of very long-chain acyl-CoA dehydrogenase deficiency in pregnancy. J Perinatol. 2010; 30: 558-62. [PubMed: 20668464]

22. Voermans NC, van Engelen BG, Kluijtmans LA, Stikkelbroeck NM, Hermus AR. Rhabdomyolysis Caused by an Inherited Metabolic Disease: Very Long-chain Acyl-CoA Dehydrogenase Deficiency. Am J Med. 2006; 119: 176-9. [PubMed: 16443431]

23. Merinero B, Pascual Pascual SI, Pérez-Cerdá C, Gangoiti J, Castro M, Garcia MJ, et al. Adolescent myopathic presentation in two sisters with very long-chain acyl-CoA dehydrogenase deficiency. J Inherit Metab Dis. 1999; 22: 802-10. [PubMed: 10518280]

24. Diekman EF, Van Der Pol WL, Nievelstein RAJ, Houten SM, Wijburg FA, Visser G. Muscle MRI in patients with long-chain fatty acid oxidation disorders. J Inherit Metab Dis. 2014; 37: 405-13. [PubMed: 24305961]

25. Laforêt P, Acquaviva-Bourdain C, Rigal O, Brivet M, Penisson-Besnier I, Chabrol B, et al. Diagnostic assessment and long-term follow-up of 13 patients with Very Long-Chain AcylCoenzyme A dehydrogenase (VLCAD) deficiency. Neuromuscul Disord. 2009; 19: 324-9. [PubMed: 19327992]

26. Goetzman ES, Wang Y, He M, Mohsen A-W, Ninness BK, Vockley J. Expression and characterization of mutations in human very long-chain acyl-CoA dehydrogenase using a prokaryotic system. Mol Genet Metab. 2007; 91: 138-47. [PubMed: 17374501]

27. Hoffmann L, Haussmann U, Mueller M, Spiekerkoetter U. VLCAD enzyme activity determinations in newborns identified by screening: a valuable tool for risk assessment. J Inherit Metab Dis. 2012; 35: 269-77. [PubMed: 21932095]

28. Miller MJ, Burrage LC, Gibson JB, Strenk ME, Lose EJ, Bick DP, et al. Recurrent ACADVL molecular findings in individuals with a positive newborn screen for very long chain acyl-coA dehydrogenase (VLCAD) deficiency in the United States. Mol Genet Metab. 2015; 116: 139-45. [PubMed: 26385305]

29. Arnold GL, Van Hove J, Freedenberg D, Strauss A, Longo N, Burton B, et al. A Delphi clinical practice protocol for the management of very long chain acyl-CoA dehydrogenase deficiency. Mol Genet Metab. 2009; 96: 85-90. [PubMed: 19157942]

30. Smelt AHM, Poorthuis BJHM, Onkenhout W, Scholte HR, Andresen BS, van Duinen SG, et al. 10.1212/wnl.44.3_part_1.467. Ann Neurol. 1998; 43: 540-4. [PubMed: 9546340]

31. Ribas GS, Vargas CR, Wajner M. 1-carnitine supplementation as a potential antioxidant therapy for inherited neurometabolic disorders. Gene. 2014; 533: 469-76. [PubMed: 24148561] 

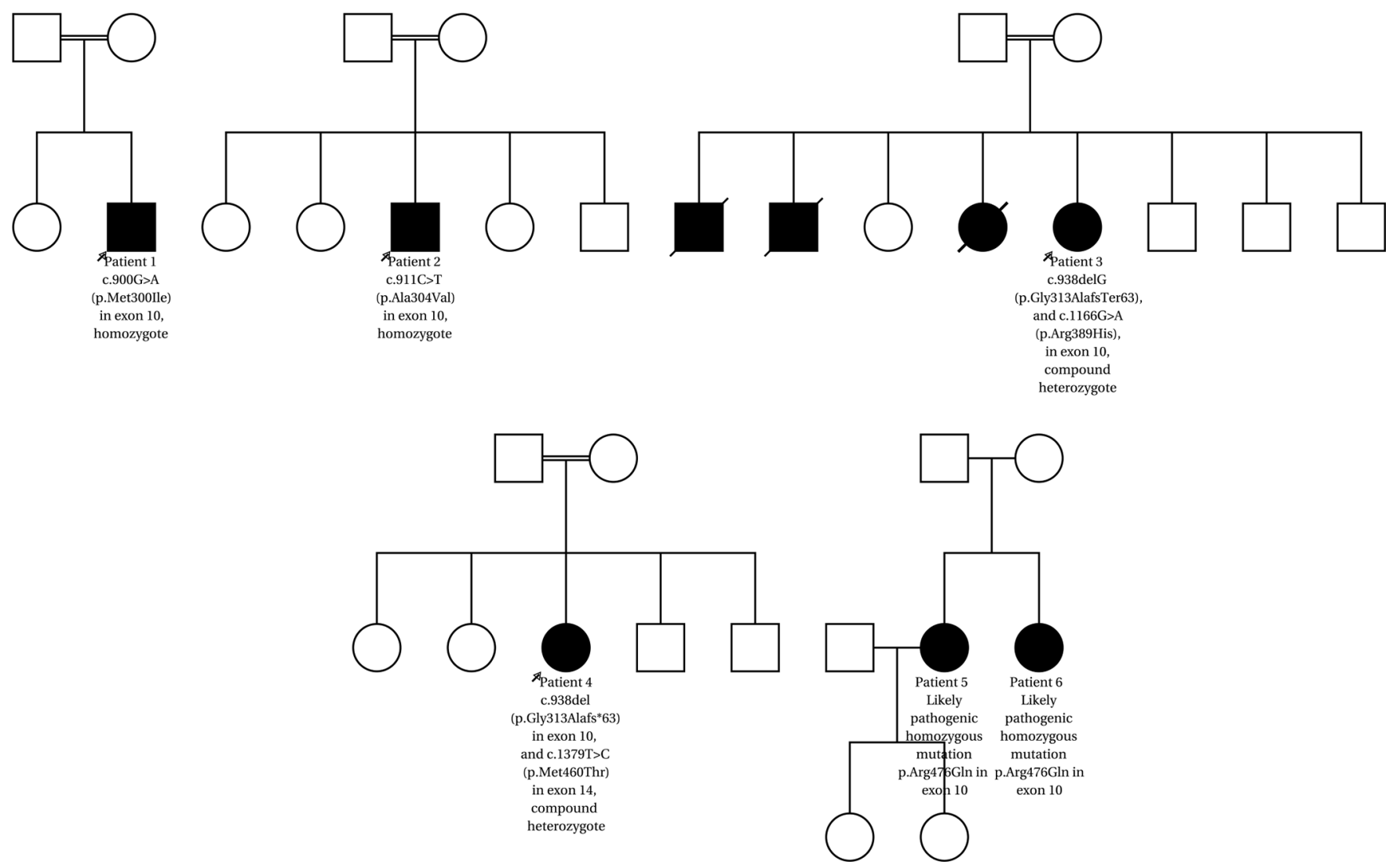

Figure 1.

The pedigree of patients 

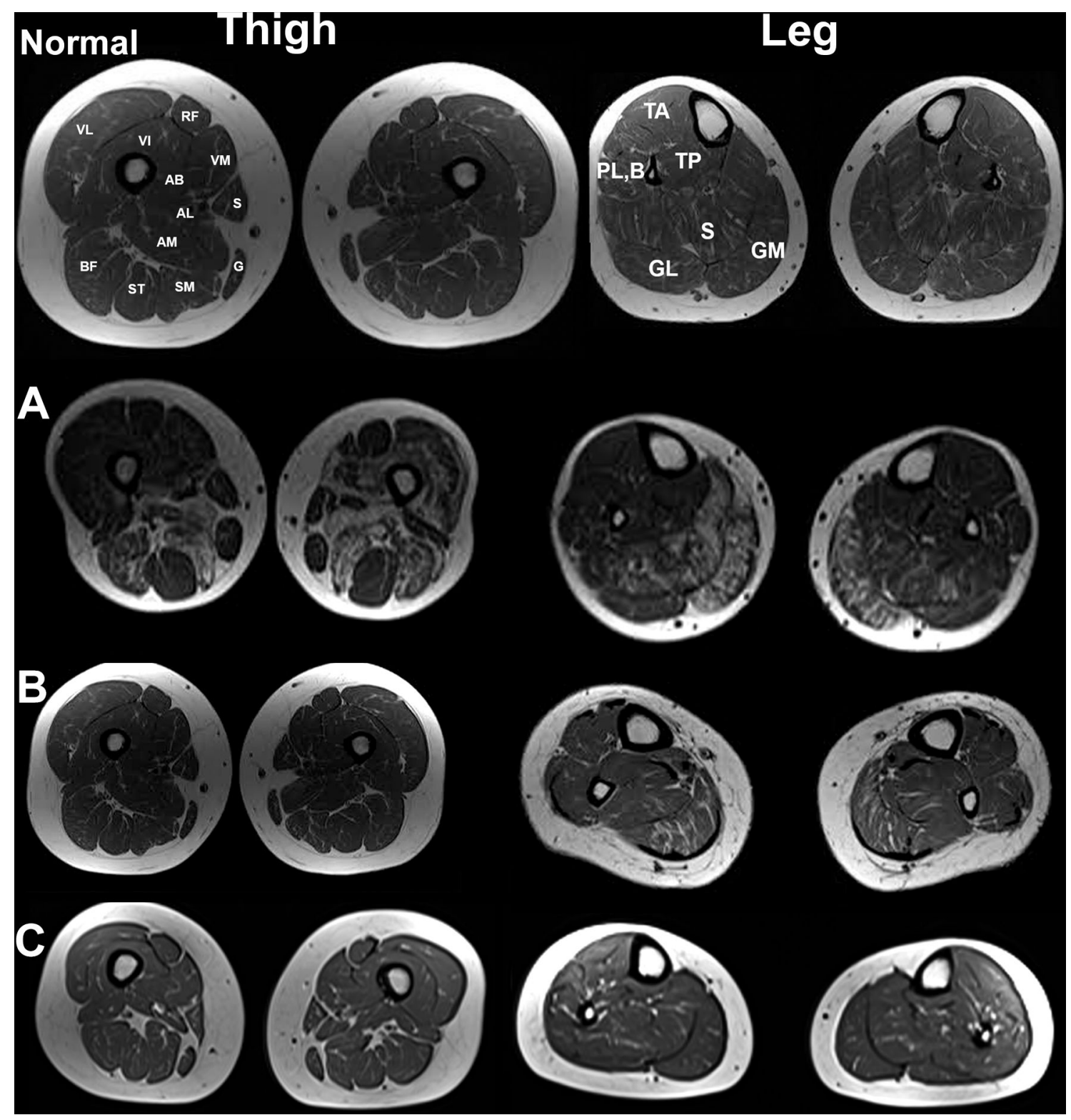

Figure 2.

Upper: Normal muscle MRI of thigh and leg. Muscle MRI of patients 1, 4, and 5. Part A: muscle MRI of the Patient1, Muscle MRI (T1 sequence) disclosed moderate deposition of fat in adductor, and vastus muscles and Sartorius, Gracilis, Rectus Femoris, and Semitendinosus muscles are spared. The findings are asymmetrical. Part B: muscle MRI of the Patient 4, leg MRI (T1 sequence) indicating the involvement of medial heads of Gastrocnemius and Soleus, and spared Tibialis anterior, Tibialis posteriors, Peroneus longus, and brevis. Part C: muscle MRI of the Patient 5, leg MRI (T1 sequence) indicating the mild involvement of medial heads of Gastrocnemius and Soleus, and spared Tibialis anterior, Tibialis posteriors, Peroneus longus, and brevis and thigh muscles. AB: Adductor Brevis; AL: Adductor Longus; AM: Adductor Magnus; BF: Biceps Femoris; G: Gracilis; GM: Gastrocnemius (lateral head); GM: Gastrocnemius (medial head); PL,B: Peroneus Longus 
and Brevis; S: Soleus; TA: Tibialis Anterior; TP: Tibialis Posterior; RF: Rectus Femoris; S: Sartorius; SM: Semi-membranous; ST: Semi-tendinous; VI: Vastus Intermedius; VL: Vastus Lateralis; VM: Vastus Medialis. 


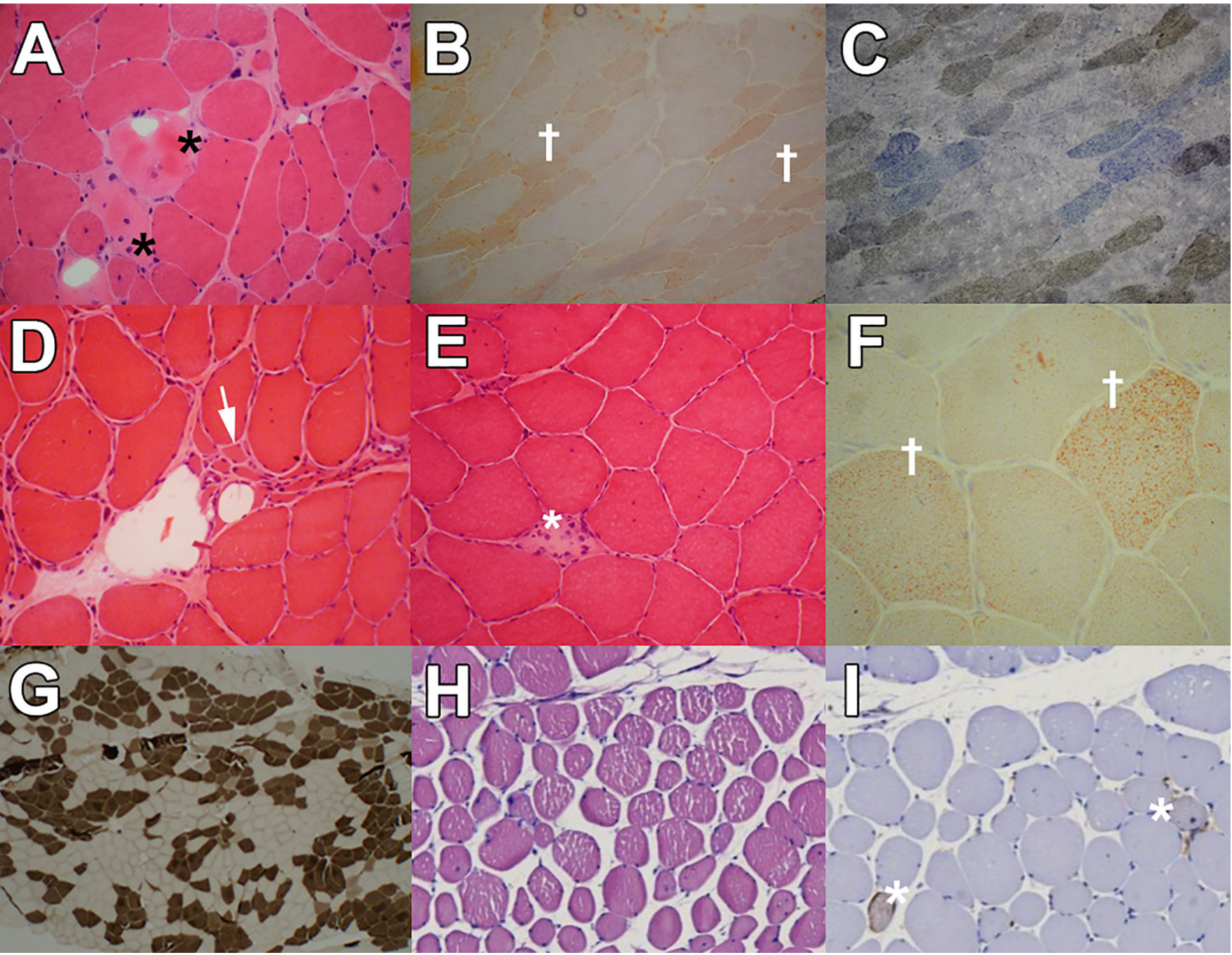

Figure 3.

Muscle pathology of the Patient 1 ( A-C): 3A: H\&E stain revealed dispersed round atrophic fibers with some necrotic $\left(^{*}\right)$ and degenerative/regenerative fibers and nuclear clumps (x400). 3B: ORO stains showed fine lipid droplets $(\dagger)$ in muscle fibers (x400). 3C: Cox + SDH stain revealed some cox-negative fibers (blue fibers) (x400). Muscle pathology of the Patient 4 (D-G): 3D and 3E: H\&E stain revealed necrotic fiber (*) with myophagocytosis and round fibers and increased internalized nuclei as well as groups of angular atrophic fibers $(\rightarrow)$ with nuclear clumps. 3F: ORO stain revealed a slight increase in the lipid content of some muscle fibers $(\dagger)$. 3G: ATPase stain (PH: 9.4) revealed prominent fiber type grouping. Muscle pathology of Patient 5 (H-I): $3 \mathrm{H}$ : H\&E stain showed variation in size and round fibers. 3I: CD-56 stain revealed some CD-56 positive fibers $(*)$. 


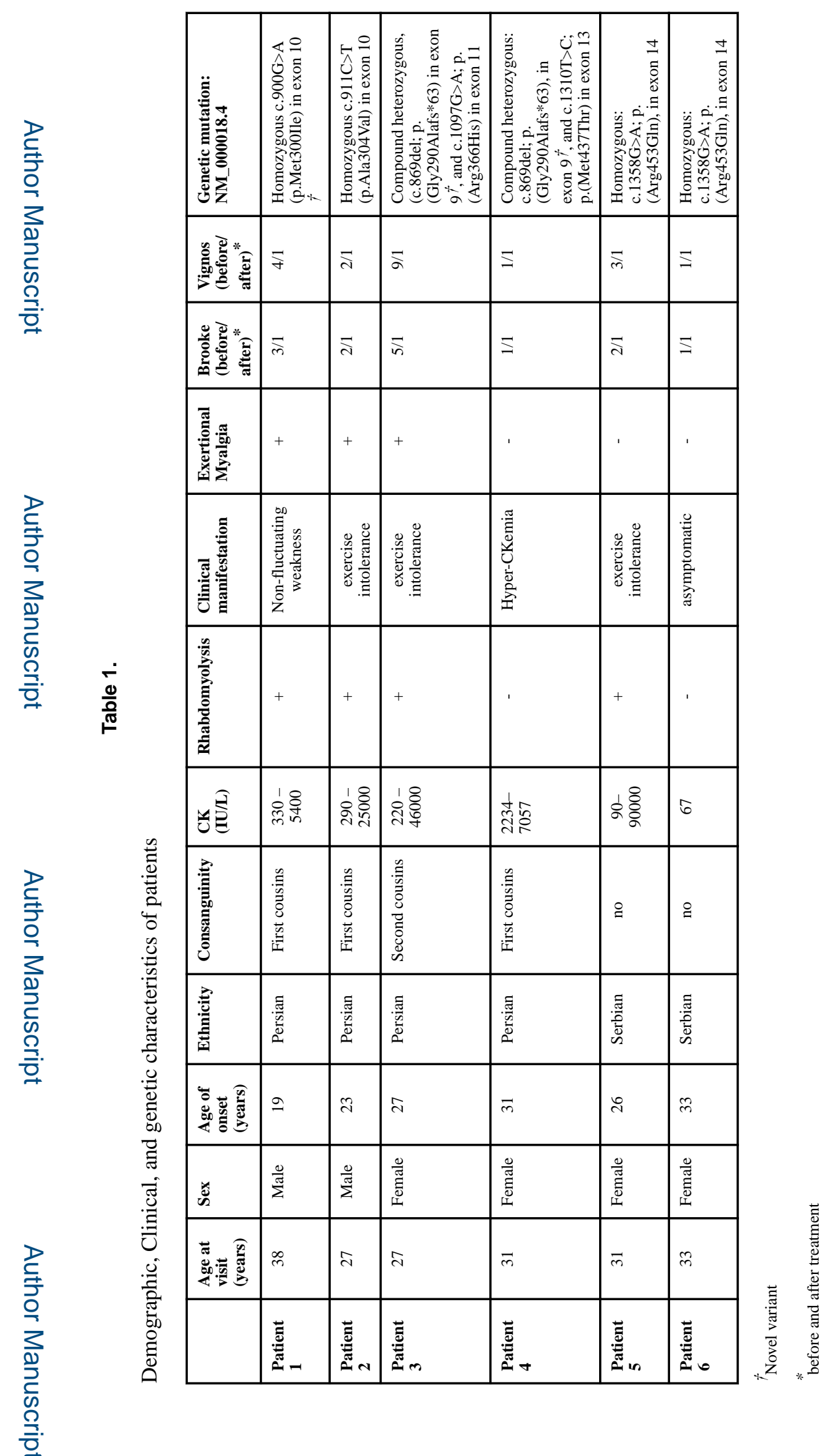

Eur J Neurol. Author manuscript; available in PMC 2021 November 01. 
Table 2.

Demographic, clinical and genetic characteristics of previously reported patients

\begin{tabular}{|c|c|c|c|}
\hline First Author & $\begin{array}{l}\text { Age of onset, } \\
\text { sex }\end{array}$ & Clinical manifestation & Genetic \\
\hline $\begin{array}{l}\text { Herrera-Olivares } \\
\text { A.M. }[10]\end{array}$ & $23, \mathrm{~F}$ & Recurrent rhabdomyolysis & $\begin{array}{l}\text { Compound heterozygous: c.589G }>\text { A (p.Val197Met) and } \\
\text { c. } 1742 \mathrm{~T}>\mathrm{C} \text { (p.lle581Thr) }\end{array}$ \\
\hline Hisahara, S.[11] & $17, \mathrm{M}$ & Recurrent rhabdomyolysis & $\begin{array}{l}\text { Heterozygous: missense mutation, c. } 1242 \mathrm{G}>\mathrm{C} \text { p. }(\mathrm{Glu} 414 \mathrm{Asp}) \\
\text { in exon } 12 \text { with single nucleotide polymorphism: a C }>\mathrm{T} \\
\text { variation in intron } 6 \text {. }\end{array}$ \\
\hline Voermans, N.C.[12] & $51, \mathrm{~F}$ & Exercise intolerance & $\begin{array}{l}\text { Heterozygous: c. } 1322 \mathrm{G}>\mathrm{A} \text { p.(Gly } 441 \mathrm{Asp}) \text {. The second } \\
\text { mutation may have been missed if it is located outside the } \\
\text { examined region of the gene. }\end{array}$ \\
\hline Smelt AH.[25] & $40, \mathrm{~F}$ & Muscle pain and stiffness & $\begin{array}{l}\text { Compound heterozygous: } \mathrm{G}>\mathrm{A} \text {; } \mathrm{p} \text {.(Gly401Asp) in exon } 13 \text { and } \\
\mathrm{G}>\mathrm{A} \text { in exon } 14 \text { (p.(Arg410His) }\end{array}$ \\
\hline Stępień K.M.[14] & $17, \mathrm{~F}$ & Rhabdomyolysis & Homozygous: c.1106T>G; p.(Phe369Cys), in exon 11 \\
\hline Murata K.Y.[15] & $29, \mathrm{~F}$ & Exercise-induced muscle pain & Homozygous: c.272C>T; p.(Pro91Leu) \\
\hline $\begin{array}{l}\text { Mendez-Figueroa } \mathbf{H} . \\
{[16]}\end{array}$ & $20, \mathrm{~F}$ & $\begin{array}{l}\text { Recurrent episodes of muscle } \\
\text { weakness, chronic myalgia, } \\
\text { rhabdomyolysis }\end{array}$ & $\begin{array}{l}\text { Compound heterozygous: c. } 848 \mathrm{~T}>\mathrm{C} \text { (p.Val283Ala) and c.879- } \\
8 \mathrm{~T}>\mathrm{A}\end{array}$ \\
\hline Voermans N.C.[17] & $20, \mathrm{M}$ & $\begin{array}{l}\text { Exercise-induced } \\
\text { rhabdomyolysis }\end{array}$ & $\begin{array}{l}\text { Heterozygous: c. } 848 \mathrm{~T}>\mathrm{C} \text { p.(Val243Ala) and c. } 1322 \mathrm{G}>\mathrm{A} ; \mathrm{p} . \\
\text { (Gly401Asp) }\end{array}$ \\
\hline Merinero B.[18] & $17, \mathrm{~F}$ & $\begin{array}{l}\text { Exercise-induced muscle pain } \\
\text { and myoglobinuria. }\end{array}$ & $\begin{array}{l}\text { Compound heterozygous: c. } 1213 \mathrm{G}>\mathrm{C} ; \mathrm{p} \text {.(Asp365His) in exon } \\
12 \text { and c. } 1349 \mathrm{G}>\mathrm{A} ; \mathrm{p} \text {.(Arg } 410 \mathrm{His}) \text { in exon } 14 .\end{array}$ \\
\hline
\end{tabular}

